\title{
A psycho-Geoinformatics approach for investigating older adults' driving behaviours and underlying cognitive mechanisms
}

Qian (Chayn) Sun ${ }^{1 *}$ D, Jianhong (Cecilia) Xia ${ }^{2}$, Jonathan Foster ${ }^{3}$, Torbjörn Falkmer ${ }^{4}$ and Hoe Lee ${ }^{4}$

\begin{abstract}
Introduction: Safe driving constantly challenges the driver's ability to respond to the dynamic traffic scene under space and time constraints. It is of particular importance for older drivers to perform sufficient visual and motor actions with effective coordination due to the fact of age-related cognitive decline. However, few studies have been able to integrate drivers' visual-motor behaviours with environmental information in a spatial-temporal context and link to the cognitive conditions of individual drivers. Little is known about the mechanisms that underpin the deterioration in visual-motor coordination of older drivers.

Development: Based on a review of driving-related cognitive decline in older adults and the context of drivervehicle-environment interactions, this paper established a conceptual framework to identify the parameters of driver's visual and motor behaviour, and reveal the cognitive process from visual search to vehicle control in driving. The framework led to a psycho-geoinformatics approach to measure older drivers' driving behaviours and investigate the underlying cognitive mechanisms. The proposed data collection protocol and the analysis and assessments depicted the psycho-geoinformatics approach on obtaining quantified variables and the key means of analysis, as well as outcome measures.

Conclusions: Recordings of the driver and their interactions with the vehicle and environment at a detailed scale give a closer assessment of the driver's behaviours. Using geoinformatics tools in driving behaviours assessment opens a new era of research with many possible analytical options, which do not have to rely on human observations. Instead, it receives clear indicators of the individual drivers' interactions with the vehicle and the traffic environment. This approach should make it possible to identify lower-performing older drivers and problematic visual and motor behaviours, and the cognitive predictors of risky driving behaviours. A better targeted regulation and tailored intervention programs for older can be developed by further research.
\end{abstract}

Keywords: Older drivers, Cognitive abilities, Visual-motor coordination, Eye tracking, Vehicle movement tracking, Psycho-Geoinformatics

\footnotetext{
* Correspondence: chayn.sun@rmit.edu.au

${ }^{1}$ Geospatial Science, School of Science, RMIT University, 124 La Trobe Street,

Melbourne, VIC 3000, Australia

Full list of author information is available at the end of the article
} 


\section{Introduction}

Epidemiological studies have projected a large increase in the percentage of drivers aged 60 and older, a cohort at risk of road crashes [1-3]. Older drivers have one of the highest vehicle crash rates in comparison with other population groups, largely due to the functional effects of the accumulation, and progression of age-related declines in visual, cognitive and motor conditions that impact on fitness to drive [4]. Knowledge of the cognitive characteristics associated with particular driving behaviour of older people has important implications for the improvement of safety on our roadways and the mobility of older adults. Safe driving constantly requires the driver's ability to response to the dynamic traffic scene under space and time constraints, the ability to perform sufficient visual and motor actions with effective coordination between the actions. This is of particular importance for older drivers who are more likely experiencing age-related cognitive declines.

Various efforts have been attempted to attain a better understanding of driving abilities, by laboratory-based neuropsychological tests, on-road driving observations, computer simulations, etc., and new methods are constantly emerging. The commonly acknowledged 'gold standard' for driving assessment is the on-road driving evaluation offering a pass or fail outcome, which is similar to the 'road test' that most individuals undergo to receive their driver's license. Although an on-road driving assessment presents a naturalistic test setting, it lacks sensitivity to detect subtle changes in driving performance when considering its utility as an outcome measure [5]. Moreover, vehicle driving in real traffic is a complex interactive system involving human visual perception, cognition, and locomotion of the driver, vehicle movement, and the environment. Driving makes demands on multiple cognitive processes for the drivers, it is challenging to generalise findings and predict safety outcomes from a single sensory tracking or an isolated assessment [6]. Thus when studying driver behaviour under actual conditions, utilising contextual driving information, drivers' actions and thinking in an integrated format should be considered. Ramon, Clarion et al. [7] developed a platform that provides a reliable data acquisition and processing solution to obtain vehicle information, video and contextual data from field tests. In their study, the driver's behaviour was assessed through various indicators, which brought abundant information to improve reliability. Although recordings across time gave a better understanding of driver's behaviour [7], Ramon's system didn't collect certain useful data to detect driver states such as visual attention measures and advanced driving variables including lane position, the nature and underlying mechanisms of the monitored behaviour hadn't been fully explored in the project publication [8].
Connections between the driver's visual actions and steering performance have been found in several studies [9-11]. For example, Land and Lee [10] made simultaneous recordings of steering-wheel angle and drivers' gaze direction during a series of driving tests along a winding road. Based on the analysis of performance on each route sections, they found that drivers relied primarily on the tangent point on the inside of each curve. The study obtained valuable information about how humans view, think and act in relation to the vehicle control and environment at the section level. Taking above studies a step ahead, fully integrating older driver's visual and vehicle control data with environmental information in a finer spatial-temporal context would give more valuable and closer assessment of driver behaviours. Especially, when many on-road driving tests failed to observe errors or mistakes by older drivers due to their driving experiences. This paper aims to develop a new approach that makes it possible to integrate multi-cognitive and multi-sensory data in a close-to-reality driving context in order to identify lower-performing older drivers and problematic visual and motor behaviours, the problematic road sections, and the cognitive predictors of risky driving behaviours.

To achieve above tasks, current paper first reviews the age-related cognitive abilities which would affect older drivers' driving abilities, followed by developing a conceptual framework to identify the parameters of driver's visual and motor behaviour, and reveal the cognitive process from visual search to vehicle control in driving. The framework leads to the propose of a new approach, psycho-geoinformatics, to measure older drivers' driving behaviours and discover the underlying cognitive mechanisms. Technically, such measurement cannot be obtained without some sort of geoinformatics tracking and analysis tools, which would synthesize multi-sensory data into a more dynamic and quantitative format and create a context-aware investigation platform. The individual driving data can be analysed in GIS (Geographic Information System) and linked to drivers' cognitive states. The psycho-geoinformatics approach serves as a platform for exploring the interactions and relationships between the drivers, vehicles and traffic environment, and determining the underlying cognitive forces leading to driving ability deterioration. The paper concludes with a theoretical discussion on the appropriateness of the proposed approach and highlights the research path moving forward.

\section{A conceptual framework of driving behavior and underlying cognitive mechanisms}

2.1 Age-associated cognitive abilities relevant to driving performance in spatial-temporal context

Among various studies, cognitive predictors of driving performance including visual processing speed and 
attention, spatial abilities, motor speed, and executive function are most studied [5]. The control of movement is a complex interaction of cognitive and sensorimotor systems. In this section, the major changes that occur in the control and coordination of movement with respect to aging are reviewed.

\subsubsection{Visual processing speed and attention}

Even in normal aging there is a decline in various aspects of visual attention including selective attention, divided attention, sustained attention and switching attention. Driving in traffic requires the ability to attend to relevant information and to ignore irrelevant information in often complex visual scenes [6]. Therefore, the speed at which visual information is processed can be critical for negotiating challenging traffic situations [1215]. It is commonly recognised that older adults are slower than younger adults in the speed of processing and that slowing of fundamental cognitive processes may have detrimental effects on more complex tasks [16]. Older adults showed a significantly decreased ability to divide attention when compared with young and middle-aged adults [17]. It would take an older driver 1.5 to 1.7 times longer on average than a younger driver to scan for information [18]. Apparently, for older drivers, visual attention ability is an important variable having a direct association with accident risk $[13,19]$.

\subsubsection{Spatial abilities}

Spatial abilities imply the generation, retention, retrieval, and transformation of visual-spatial information [20]. General spatial abilities, which peak during the second or third decades of life, decline steadily in later life. Older adults experience difficulty with spatial relations and with mental rotation tasks, for example, localizing objects in 3-D space and interpreting information displayed in the rear view and side mirrors of a car [18]. Previous studies have linked spatial cognition to older drivers' driving behaviours [20-22]. A review of older drivers' capabilities for safe driving [22] indicated that spatial cognition is frequently utilised in driving and related to the safe and efficient operation of a vehicle.

\subsubsection{Psychomotor skill}

Motor skills in driving, such as pedal control and steel wheel control are psychomotor skills developed as a result of constant practice, so individuals need little thought to perform them but require input from both the physical and the mental attributes, and coordinate in order to achieve a certain goal. Age-related motor skill declines can be found as balance and gait deficits, coordination deficits, movement slowing [23], and alterations in cognitive-motor processes [23]. Motor execution of the older adults may require more psychomotor ability and rely on cognitive control [24].

Poor movement skill in older adults results in reduced speed and accuracy [25]. Older drivers are slower to respond to situations [18], e.g., break reaction time, which affects ability to respond quickly to imminent danger; reduced control, which impacts safe driving in a timely fashion. Older adults take longer to initiate movement and carry through and are slower to accomplish eye movements to fixate on objects that are moving around the environment [18]. Older adults tend to adopt a different movement strategy when facing with a motor task that requires them to move steadily under temporal and/or spatial task constraints [12].

\subsubsection{Executive function}

Neuroimaging studies showed age-related changes in the prefrontal cortex which indicate the decline of executive function in normal aging [26]. Executive function is necessary for integrating information and making decision, which seems highly relevant to driving [12]. Executive function involves the control and coordination of cognitive operations [27]. Subtle executive changes in a more cognitively intact group (such as older people), show a stronger relationship with cognitively demanding driving tasks [28]. The extent to which visual perception and fine motor skills are well coordinated is critical in driving [5].

Executive function handles organizing actions in goal-oriented behaviors, specifically coordinating different centers of the brain to respond to environmental cues, planning the response and subsequently carrying it out, and dealing with situations that are hazardous or technically challenging [29-31]. Given that crashes in older adults involved often occur in complex traffic situations, such as intersections [32], it is reasonable to assume that difficulty occurs at the higher cognitive level of executive function [12] in older drivers.

\subsection{The context of driving assessment in older adults}

Since cognition and behaviour are often situational in context, laboratory findings, particularly concerning decision-making and related executive functions, may fail to predict behaviours in complex and dynamic tasks that people confront in their daily lives. Vehicle driving in real traffic involves human visual perception, cognition, and locomotion of the driver, vehicle movement, and the environment [33]. The driving environment can greatly influence a driver's ability on the road. Age-related declines in the required component functions (vision, cognition, sensorimotor) for driving may often be "overloaded" in certain contextual situations [34]. Recent studies have emphasized an integrated approach to driver safety study which focuses on the 
overlapping and interacting area of the role of driver, vehicle and road environment [35]. To detect the driver state, various overt and covert measures, such as driving performance, visual attention, and traffic situation can be collected and interpreted in the driving context [8]. As movement does not take place by itself, gathering information on the context in which the movement occurred, such as the interactions of moving phenomena with their environment and with other individuals, can facilitate a deeper understanding of movement [36].

\subsubsection{The driver-environment interaction}

To drive safely, drivers need to perceive and interpret the relevant objects and elements in the current traffic situation so that they can consider these elements during planning and controlling their behaviour. Such elements may be other cars, the condition of the street or traffic signs [30]. For each of these elements, drivers not only must perceive them but also understand them according to their relevance to the driver's goals. In addition, drivers must make assumptions about the future actions or states of these elements [30]. The concept of situation awareness has become very popular in psychology as well as in driving studies to describe these cognitive processes [37]. Situations require a driver to adapt his/her behaviour by changing speed or dangerous direction. Safer drivers should be able to anticipate these situations in order to take vehicle control actions to avoid a collision [38]. Endsley [39] stressed that the concept of situation awareness is best seen as encompassing perceptual and comprehension processes, but not decision-making and response execution processes.

\subsubsection{The driver-vehicle interaction}

Once in motion, the driver must continuously and actively make adjustments on the steering wheel and pedals to attain desired travel objectives, and to avoid dangerous situations such as driving off the road or losing control of the pedals [40-42]. Driving can be described as a control task in a changing environment "created by the driver's motion with respect to a defined track with static and moving objects" [42]. The task includes requirements for route choice and following, coordination of manoeuvres in support of navigational objectives, and ongoing adjustments of steering and speed [42]. Control theory is predicated on the assumption that driver control actions are dependent on perceptual processes which select information compared to some standards. Drivers act to keep resulting discrepancies within acceptable limits in a negative feedback loop as the means of control in their goal-directed behaviour [42]. Target variables of control theory can be space and time margins and mental load specifically relating to control [43].
Smith [44] described the interacting phases in driving: first, to sense a situation and stimulus registered at the perceptual level; second, to recognize it and stimulus recognition at the cognitive level; then to decide how to respond at cognitive level; and lastly to execute the manoeuver at the motor level. Inputs from sensory and cognitive processes are important in determining what a driver choose to do and how the movements are organized and adjusted. Therefore, in driving assessments, environmental conditions, task requirements, and persons' characteristics imposing spatial and temporal constraints must be sensed and evaluated to determine what have been done [45].

\subsection{A conceptual driving behaviours and cognitive processes framework}

Driving is a complex system in which the environment, driver and vehicle are influencing factors [46]. The system incorporates drivers' visual patterns, the attribute of traffic environment and vehicle movement that are intertwined in space over time. The human-machine-environment framework (Fig. 1) links the cognitive variables and driving parameters, and various interactions associated with driving psychology and behaviours in a circular hierarchical structure.

The center of the conceptual framework (Fig. 1) abstracts the core of driving as driver-vehicle-environment interactions and where driving research should start from. The second circle (yellow) defines the cognitive variables with respect to the sub-sections of interactions. The driver's perception, visual attention, spatial orientation and spatial visualization are the critical cognitive variables for driver-environment interaction; whereas psychomotor skill and executive function are the key cognitive abilities for driver-vehicle interaction. The situation awareness theory and driver control theory are used to support the notions of the two interaction phases. In driving, the two phases are instantaneously connected through the driver's cognitive processes, which are demonstrated as the driver's visual search and locomotion in the next circle of drivers' behaviours. The third circle (blue) defines key parameters of driving behaviours and performance, and their linkages to the driver's cognitive conditions. Eye fixation and duration, steel wheel and pedal controls are the variables of drivers' main behaviours and performance.

The outer circle (grey) presents study variables of drivers' behaviours and performance. They are conceptualised as the outcomes of psycho-spatial-temporal interactions on: what and how long drivers viewed their surroundings, how gazing behaviour associated with vehicle control (e.g., lane keeping and speed regulation). The links between grouped variables give indications on the underlying neuropsychological mechanisms responsible 


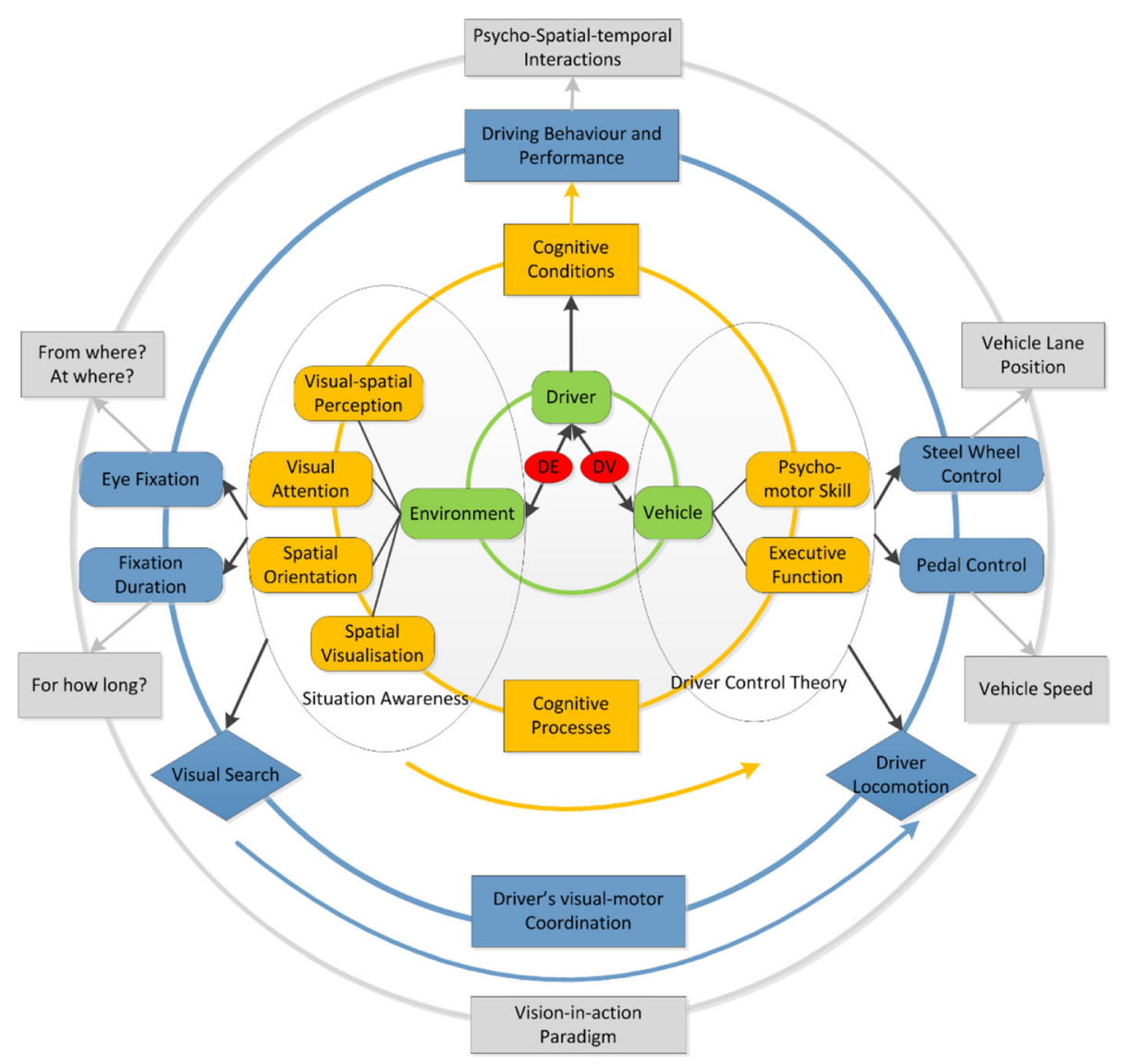

Fig. 1 A conceptual driving behaviours and cognitive processes framework

for driving behaviour and performance, such as the driver's ability of visual-motor coordination determines the driver's locomotion, and results in the vehicle lane position and the speed. The preliminary implication for older adults is that variability in the performance of older drivers may stem from age-related declines in cognitive functioning, and we must go beyond single risk factor studies of crashes to identify the factors most highly related and predictive of safe driving [47]. An important further implication of this framework is that research focus should be directed towards unpacking older drivers' visual-motor coordination using a vision-in-action paradigm in order to determine the underlying cognitive mechanisms.

\subsection{Unpacking visual-motor coordination in older drivers}

The conceptual framework has emphasised that driving involves multiple tasks that require drivers to gather information and control the vehicle to achieve desired goals. In other words, drivers face coordination demands arising from the dependencies between sub-activities that constrain how tasks can be performed. Therefore, coordination problems should be managed by sub-activities that implement coordination effectively. A driver's visual and motor behaviour must be coordinated, and how well it was coordinated determines the driving ability and indicates the level of the driver's mobility and safety. When applying visual-motor coordination concept in a driving behaviour study, it is important to include key sub-activities in the data model. Therefore, driving assessment or training should always consider how overall goals can be subdivided into tasks so that the coordination can be improved.

Previous studies show that older drivers are less likely to be involved in crashes caused by fatigue, high speed, weather condition or alcohol, but are more likely to be involved in crashes involving: intersections; failure to yield the right of way; failure to identify hazards or to heed stop signs/traffic signals; turning and changing lanes [32, 48, 49]. Older drivers also tend to avoid driving in a busy traffic or at night, and especially at nights in wet conditions, however, older drivers are more likely to be involved in angled impact crashes [50]. It is more difficult for older drivers to gather and process information about the environment due to the gradual deterioration of sensory and cognitive processing capabilities 
[51]. According to Michon [52], there are three levels of hierarchical decisions which shape driving behaviours: strategic-level decisions such as route selections; tactical-level moment-to-moment decisions such as speed choice and lane changing; and operational-level second-to-second behaviour such as braking or steering. Older drivers are more capable to perform their strategic and tactical decisions than the operational-level ones, which involve quick vehicle handling to implement the decisions at the tactical-level. It is performed almost without conscious thoughts [53], thus requires effective executive functioning and coordination between actions. In general, visual-motor coordination can be construed as the extent to which visual perception and fine motor skills are well coordinated [54]. Older drivers have exhibited difficulty in adjusting their operational-level behaviours, consequently they would have shown problems in visual-motor coordination [55]. The investigation of cognitive mechanisms related to older drivers' behaviours has been an area of high priority due to the increasing elderly population, the visual-motor coordination can be a sensitive but reliable measurement for the prediction of driving ability among older adults. On the other hand, older drivers must be aware of their abilities and limitations in visual-motor coordination so that they can compensate by employing defensive visual search and lane keeping behaviours to enhance their driving competencies.

\section{A psycho-Geoinformatics approach for driving and visual-motor coordination assessment}

Adapting tools and techniques from the information technologies can help improve the measurement and modelling of psychological processes in a broader range of ways. In fact, no matter how data are to be obtained, they will be synthesized by time or location during analysis, so that researchers can get a sense as to what happened in the driving scene and context. Psycho-Geoinformatics, which we propose in this paper, can achieve such a goal since it contains: 1), rich data sources from mobile devices and sensors; 2), a central data store; and 3), an analytical platform for investigation [56,57]. With the availability of numerable human movement data, the constantly growing movement of users (such as drivers) with "wearable" equipment will offer a great potential for more disciplines "as psycho and physiological parameters could be collected on the go" [58]. A studied context with more detailed spatial-temporal scale can reveal subtle changes in actions which suits driving behaviour tracking and unpacking visual-motor coordination, as the fact that both visual search and vehicle control actions update from moment to moment at the micro scale level, for example, the driver's eye fixation duration can be as short as $0.1 \mathrm{~s}$. For such reason, the data collections through driving experiments and the analysis of visual-motor coordination require advanced tracking technologies and methods of data integration.

\subsection{Data collection protocol}

Based on the driving behaviour variables identified in the conceptual framework, a data collection protocol (Fig. 2) is presented as part of the psycho-geoinformatics solution. A variety of older drivers' attributes and driving behaviours are to be captured in one investigation through both the laboratory neuropsychological tests (left) and the on-road driving assessment (right). The laboratory neuropsychological tests can provide a window into the specific cognitive deficits in individuals with increased crash risk. The presented neuropsychological tests in Fig. 2 have been described in our previous paper [59]. Such testing is typically sensitive to gross

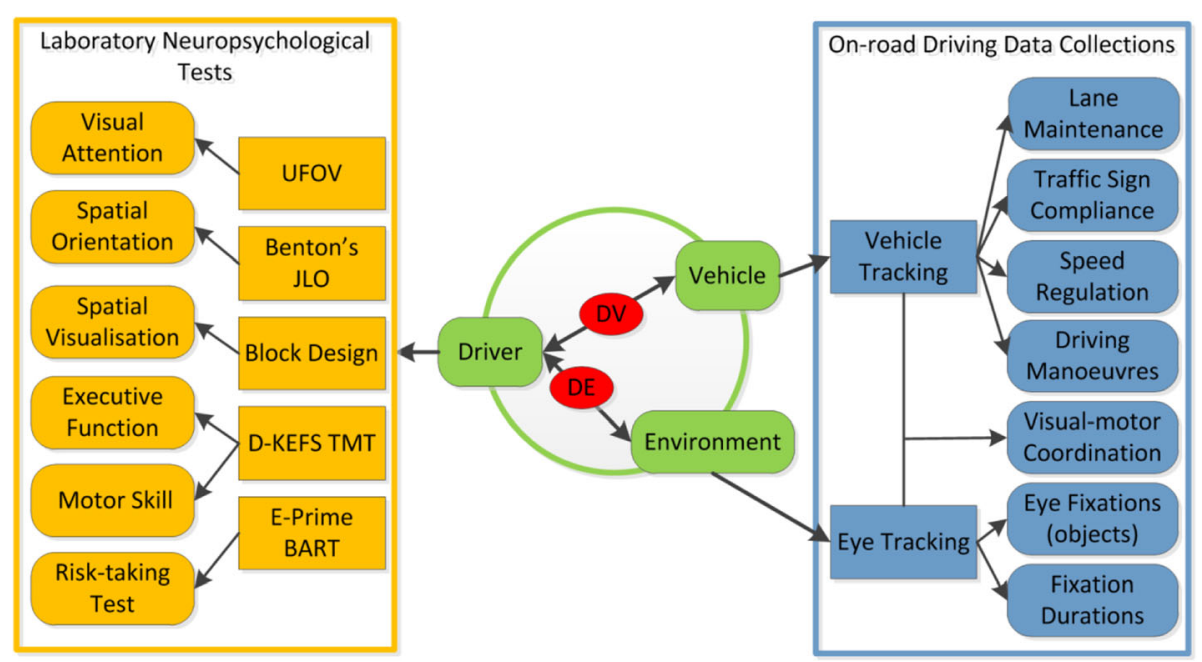

Fig. 2 Data collection protocol - laboratory and on-road assessment 
abnormalities and is often used to assess cognitive deficits in the clinic [6]. Studies have shown relationships between the performance of at risk drivers on laboratory neuropsychological tests of cognitive function and driving behaviour both in simulation and on road driving [60], with a number of studies showing the predictive value of neuropsychological tests on driving behaviour in healthy aging [61]. Nevertheless, the direct link between drivers' cognitive conditions and driving performance can be hardly established without detailed driving data.

Vehicle kinematic profiles in common roadway scenarios, such as the lane position and speed, may provide specific evidence of driving behaviour in older drivers with age-associated declines in visual, cognitive, and attentional impairments that relate to overall driving safety and cognitive abilities. Our earlier studies have showed the possibilities of vehicle movement tracking and the definitions of the vehicle control variables [59, 62]. Using such data collection for at-risk drivers is likely to provide an abundance of knowledge and a context of risky behaviours. This data can also verify the assumptions of theoretical models and the results of laboratory studies regarding the role of variables such as reaction time, or lane deviation [6], and provide evidence on how cognitive abilities affect driving behaviours [59].

Eye tracking is one widely used technique to measure eye movement so that researchers can find where the person was looking at a given time, and the sequence of eye movement shifting from one location to another [63]. The rationale behind eye tracking research was depicted by Tatler [64] that human eyes are directed to the locations that are relevant to our task at each moment. We look at the locations that convey information allowing us to act upon the environment in order to complete our current motor action. When and where drivers look at is of importance to driver safety $[65,66]$. The main parameters for visual search behaviour are the frequency and the duration of eye fixations, which are the time periods where the driver's gaze locations were spatially relatively stable. Analysis of eye fixations facilitates the investigation of the driver's visual awareness and driver behaviours in dynamic driving situations $[67,68]$.

The on-road driving tests can simultaneously record the vehicle movement and the driver's eye movement. The driving route should contain roundabouts and intersections, which are defined as critical sections for analysis. This is based on the finding that the high vehicle crashes involvement of older drivers occurred at those sections $[32,48,49]$. A head-mounted eye tracker records eye fixations and duration on video images for the analysis of the driver's visual pattern. A GNSS (Global Navigation Satellite System) device on the driver's car roof records the vehicle position every $0.1 \mathrm{~s}$. RTK
(Real-time Kinematic) technique for vehicle positioning can be used to achieve centimetre to decimetre level accuracy $[62,69]$. This is essential to calculate the vehicle position and speed so that the performance level of vehicle control in individual older drivers can be quantified $[62,69]$.

The data collection protocol employs a vision-in-action paradigm in an attempt to integrate visual-perception data with motor action data. Such a paradigm recognises many factors in the constraints-directed model: the driver, the task, the environment and perception-action coupling within driving visual-motor workspaces. To integrate the data, the eye fixations are geocoded and synchronised with the vehicle movement trajectory in a GIS program [70] so that the researchers are able to view drivers' visual-motor behaviours retrospectively and conduct spatial and statistical analysis [71].

\subsection{Analysis and assessments framework}

The analysis and assessment framework (Fig. 3) outlines spatial and statistical approaches for assessments and expected outcomes. Technically, it establishes an analytical platform of psycho-geoinformatics to investigate the driving behaviours and the cognitive mechanisms.

A central driving geodatabase (Additional file 1: Appendix) can be created to store and integrate spatial-temporal data of vehicle trajectory and eye movement parameters. The drivers' laboratory neuropsychological scores are linked to driving the performance database (Additional file 1: Appendix). Various spatialtemporal analysis and statistical analysis can be then performed across the datasets. Four subsets (Fig. 3) of analysis and assessments encompass examples of our earlier work and potential future work. First, the driving assessment evaluates driving competencies with respect to the level of safety, efficiency, and workload. The performance ranking models and benchmarks the visual-motor coordination performance, and identifies the underperforming older drivers. The spatial-temporal patterns of drivers' eye movement and vehicle control parameters can be visualized at both individual and group level to find the distributions of visual attention and vehicle control. Finally, a range of statistical analysis and modelling can be performed to investigate correlations and predictive relationships between driving behaviours and cognitive abilities.

\subsection{Driving competency assessment}

The quantitative eye movement and vehicle control data can be used to assess individual drivers' driving competency in terms of visual workload, vehicle control efficiency and safeness, which cover the three essential aspects of driving performance. The driver's gaze effort can be used to measure the level of the workload during driving. In a particular manoeuvre, the scale of workload 


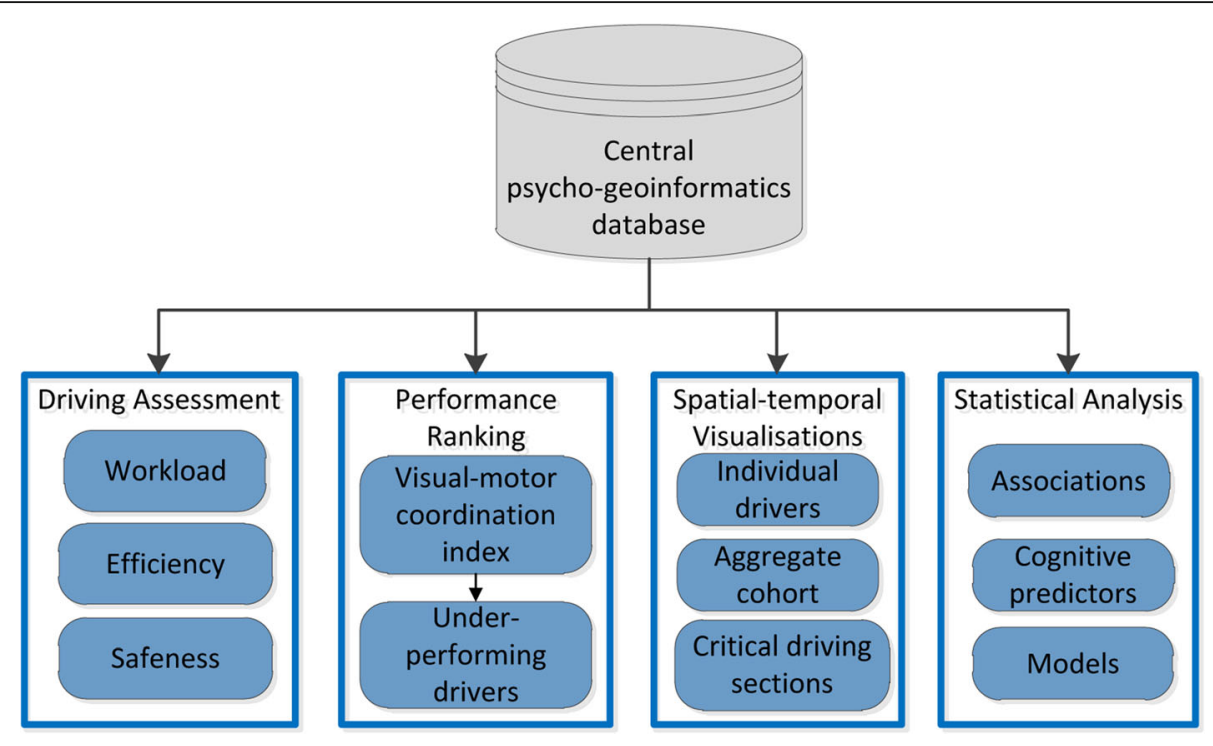

Fig. 3 Analysis and assessments framework

can be calculated by the ratio between total gaze effort (number of eye fixation objects*number of eye fixations"durations) and total manoeuvre time. The driving competencies of efficiency can be calculated by the lane deviation of vehicle trajectory from the desired path (e.g. road centreline). For the matter of safeness, we can combine the driver's vehicle speed and lane position performance by taking the consideration that they are two of the many possible factors that lead to crashes on road [72].

\subsubsection{Performance ranking: Visual-motor coordination index} Multiple parameters of visual search and vehicle control can be aggregated or modelled into a single visual-motor coordination index for overall performance ranking. A simple visual-motor coordination ranking method was presented in our publication [70]. The results gave performance scores of individuals to identify problematic behaviours for low-performing older drivers. The index can be combined with road data in GIS to inspect visual-motor coordination at different route sections, and trace back the specific road sections where drivers performed high visual workload, inefficient or unstable vehicle control. The visual-motor coordination has emerged as an important indicator of driving behaviour and performance in older drivers. The visual behaviour-vehicle control relationships described in the publication [73] provide an in-depth understanding of the difference between good and low-performing older drivers. Spatial ability, executive function, and selective attention were identified as the best predictors of visual-motor coordination performance in older drivers. They complemented the visual-motor coordination model of driving by explaining the underlying neuropsychological mechanisms. The visual-motor coordination index model can be further improved by weighting the impact of visual and motor parameters.

\subsubsection{Spatial-temporal visualisations}

The synchronised visual search and vehicle trajectory data enables spatial analysis and visualisation to examine drivers' driver-vehicle-environment interactions. The integration of high-resolution vehicle movement trajectory with other spatial information, such as roads, allows researchers to segment, visualise and model their data based on location and time attributes. The eye fixations of eye movement can be geocoded and linked to the vehicle movement trajectory to represent the visual-motor coordination of drivers in a GIS platform. The techniques were described in paper [74], where a spatial-temporal data model of visual-motor coordination was constructed. The integration extended the dimensions of eye movement tracking by adding the location of where each gaze originated and visualising the pattern of their oculomotor behaviour. A further publication [75] investigated the associations between older drivers' visual capacity and their eye fixations in various driving manoeuvres. The influence of different driving sections on the associations between older drivers' eye movement and visual ability were explored via spatial analysis and visualisations $[74,75]$.

\subsubsection{Statistical analysis and modelling}

The psycho-geoinformatics approach can be used to investigate driving behaviors and underlying cognitive mechanisms using a range of statistical analysis and modelling methods. The research questions or expected 
outcomes can be: (1) the correlations between specific measures of older drivers' driving behaviours and performance and human factors/functional ability measures, the explained variance of these relations to determine the strength of these correlations [59]; (2) the predictors of neuropsychological tests for visual-motor coordination performance [73]; (3) the discriminant variables associated with safe driving behaviours in older adults [59]; (4) a series of analyses in particular visual-motor coordination behaviours focusing on specific hypotheses and sub-scenarios; and (5) performance benchmarking models for identifying low-performing older drivers and their problematic driving behaviours [70].

\section{Discussion and prospects for future work}

Road safety studies traditionally use source data from crash reports, which are limited in the depth and quality of "real-world" information about individual drivers' behaviour and performance. On-road driving assessment can provide the opportunity to study what drivers do and how they handle a broad range of elements in real traffic: the cars, the road infrastructure and road regulations. These assessments can shed light on how different drivers interact with their vehicles and the environment, how they gain information to avoid hazards and how they adapt their visual resources to analyse increases in traffic demand [60]. Collecting comprehensive on-road driving data also represents a significant evolution in driving research, because the gaze and motor characteristics observed under laboratory conditions by driving simulation may not provide an accurate reflection of a participant's natural behaviour, and investigations conducted in a real setting may reveal important mechanisms involved in their behaviours.

On-road driving assessments have been detecting the errors made by the drivers. However, many older drivers are highly experienced in driving and hardly make obvious mistakes within the assessment period. Rather, they may demonstrate subtle changes in specific measures of driving behaviour, such as lane deviation and insufficient eye gazing, which are difficult to observe. A standardised on-road driving assessment with a quantifiable score would allow for a greater objectivity in detecting risky behaviours and evaluating the level of driving performance [61]. So far, few studies have been able to scrutinize detailed individual driving behaviour due to the lack of reliable data and available technologies. Despite an increasing amount of research on older drivers' safety and mobility, little quantitative evaluation has been done to investigate their visual-motor coordination performance and cognitive predictors for risky driving behaviours. The proposed psycho-geoinformatics approach has established a holistic platform to explore a variety of older drivers' attributes and driving behaviour in a single integrated experiment. As a result, a broad set of measures from multiple domains including driver, vehicle, and road environment in the same time period can be obtained. The integration of the driver's visual patterns, vehicle movement and the traffic can not only provide insights into the driver-vehicle-environment interactions in space over time, but also help discover the underlying cognitive processes. The framework is driver centric on an individual basis and the analysis and assessment are based on a scalable multi-dimensional data infrastructure, which is more plausible with respect to the real driving context.

The framework links between the driver's visual and motor behaviour and the cognitive process using spatial technologies. It highlights location and time as essential parameters in driving studies, and synthesizes multi-sensory data into a meaningful form so as to create a context-aware investigation platform. Such measurement cannot be obtained without geoinformatics tracking and analysis tools, when it comes to the practice of intervention, the assessment feedback for participants can be significantly cheaper than personal interaction with a therapist. The synchronised visual attention and driving performance over high resolution of space $(\mathrm{cm})$ and time (seconds) can provide researchers and practitioners a new tool to quantify the behaviour and performance.

The potential uses of the psycho-geoinformatics approach are plentiful as the application is adaptable and customisable. The approach is grounded in empirically-based research and the methods can be replicated by other researchers and practitioners. The framework and data model can be directly used or modified in: studies of other cohorts, such as novice drivers, drivers with dementia or other age-related diseases; studies for other research questions, such as distraction in driving behaviour, alcohol/ drugs effect in visual-motor coordination in driving; and studies for modelling driving behaviours and scenarios, such as driving on freeways, bus drivers' behaviours. Since the driving experiment and data analysis are capable to generate a driving matrix to quantitatively evaluate the levels of driving performance in older adults, insurance companies, such as car insurance can refer to the result in deciding the level of insurance cover for older drivers.

Another short-term application is to design a driving program to evaluate and improve older drivers' situation awareness in distracted driving. The program can record the visual search strategies and vehicle control behaviour when the older drivers are distracted by visual stimulus. The goal of such application is to provide an effective cognitive intervention program for older drivers, in order to preserve their ability to stay mobile by driving and improve the safety of these vulnerable road users.

Currently the trends of "shared mobility" and "autonomous vehicles" are still in the developing stage with 
limitations in the applications, and are more to research before both can be applied to help older people's mobility. In terms of the technology of "autonomous vehicles", the topic area of cognitive attention is in fact becoming even more important with the advent of automation of the driving task. When the vehicle is operating under its own control, it is arguably safe for the human in the vehicle to shift attention away from driving and the traffic situation. But some automation designs still require supervisory control by the human and readiness to take over at short notice [76]. Therefore, some level of attention to the external road and traffic scene, and effective visual-motor coordination are still needed and critical for older people. To make an autonomous vehicle more cognitive and artificially intelligent, it needs the system implemented using certain advanced cognition theories [77], such as situation awareness theory, visual-motor coordination theory which we have described in this paper. We believe that one of the challenges of autonomous vehicles will be to imitate human behaviour in driving interactions [77]. This probably will create a long-term application for psycho-geoinformatics approach in driving research, for example, a psycho-spatial-temporal algorithm may be developed and integrated into the driverless car system architecture.

We understand that subtle changes in drivers' behaviour lead to accidents, and for older drivers, the changes need to detect as early as possible in order to provide intervention. The presented psycho-geoinformatics approach sets up a scalable multidimensional data infrastructure which can offer significantly more definitive information related to the driver's visual search strategies and motor actions. Driving events recorded in a second-to-second level exhibit spatial distribution and temporal change of vision and motor behaviours of the driver, thus builds a detailed model for the cognitive processes of the individual drivers and their interactions with the traffic. From there, the driving competencies can be accurately evaluated in the system and potentially the system can provide effective feedback to the drivers for intervention. In conclusion, this paper has set out a feasible approach with valid theoretical and methodological framework as a basis for others to adapt and develop in exploring the complex driver-vehicle-environment interactions and cognitive mechanisms. We expect that an analysis of the relationships outlined in our conceptual framework by one of the suggested approaches in the analysis and assessment will enrich the research debate on driving behaviour with constructive insights.

\section{Additional file}

Additional file 1: Appendix. (TIF $2140 \mathrm{~kb}$ )

\section{Acknowledgements}

Thank for the Australian Postgraduate Award (APA), Curtin University Postgraduate Scholarship, Curtin University Mobility scholarship, Curtin University publication scholarship, Curtin University PhD completion scholarship, and a number of conference travel grants, for enabling Qian (Chayn) Sun's PhD research. The authors would like to also thank the anonymous reviewers of this paper for their valuable comments.

\section{Publisher's Note}

Springer Nature remains neutral with regard to jurisdictional claims in published maps and institutional affiliations.

\section{Author details \\ ${ }^{1}$ Geospatial Science, School of Science, RMIT University, 124 La Trobe Street, Melbourne, VIC 3000, Australia. ${ }^{2}$ School of Earth and Planetary Sciences, Curtin University, Perth, Australia. ${ }^{3}$ School of Psychology and Speech Pathology, Curtin University, Perth, Australia. ${ }^{4}$ School of Occupational Therapy and Social Work, Curtin University, Perth, Australia.}

Received: 19 October 2016 Accepted: 20 July 2018

Published online: 07 August 2018

\section{References}

1. Aksan $\mathrm{N}$ et al (2012) Cognitive functioning predicts driver safety on road tests 1 and 2 years later. J Am Geriatr Soc 60(1):99-105

2. Lyman $S$ et al (2002) Older driver involvements in police reported crashes and fatal crashes: trends and projections. Inj Prev 8(2):116-120

3. Evans $L$ (2000) Risks older drivers face themselves and threats they pose to other road users. Int J Epidemiol 29(2):315-322

4. Molnar FJ et al (2007) Acceptability and concurrent validity of measures to predict older driver involvement in motor vehicle crashes: an emergency department pilot case-control study. Accid Anal Prev 39(5):1056-1063

5. Schultheis MT, Manning KJ (2011) Chapter 10 - Neuroscience and Older Drivers. In: Porter BE (ed) Handbook of Traffic Psychology. Academic Press, San Diego, pp 127-136

6. Lees MN et al (2010) Translating cognitive neuroscience to the driver's operational environment: a neuroergonomic approach. Am J Psychol 123(4):391-411

7. Ramon C et al (2008) An integrated platform to assess driver's physiological and functional states. In: Engineering in Medicine and Biology Society, 2008. EMBS 2008. 30th Annual International Conference of the IEEE

8. Park S, Son J (2010) Implementation of a driver aware vehicle using multimodal information. In: 17th World Congress on Intelligent Transport Systems. Busan, South Korea

9. Land MF (2006) Eye movements and the control of actions in everyday life. Prog Retin Eye Res 25(3):296-324

10. Land MF, Lee DN (1994) Where we look when we steer. Nature 369(6483): $742-744$

11. Cloete $S$, Wallis $G$ (2011) Visuomotor control of steering: the artefact of the matter. Exp Brain Res 208(4):475-489

12. Anstey $\mathrm{KJ}$ et al (2005) Cognitive, sensory and physical factors enabling driving safety in older adults. Clin Psychol Rev 25(1):45-65

13. Richardson ED, Marottoli RA (2003) Visual attention and driving behaviors among community-living older persons. J Gerontol 58(9):832-836

14. Owsley C (2013) Visual processing speed. Vis Res 90:52-56

15. Ball KK (2003) Real-world evaluation of visual function. Ophthalmol Clin N Am 16(2):289-298

16. Glisky EL (2007) Changes in cognitive function in human aging. In: Brain aging: Models, methods, and mechanisms. CRC Press, Boca Raton, pp 3-20

17. Ponds RW, Brouwer WH, Van Wolffelaar PC (1988) Age differences in divided attention in a simulated driving task. J Gerontology 43(6):P151-P156

18. Smither JAA et al (2004) Aging and driving Part I: Implications of perceptual and physical changes. In: Human Performance Situation Awareness and Automation: Current Research and Trends, pp 315-319

19. Owsley C et al (1991) Visual/cognitive correlates of vehicle accidents in older drivers. Psychol Aging 6(3):403-415

20. Colom R et al (2002) Vehicles of spatial ability. Personal Individ Differ 32(5): 903-912

21. Anstey KJ et al (2012) The role of cognitive and visual abilities as predictors in the multifactorial model of driving safety. Accid Anal Prev 45(0):766-774 
22. Eby DW et al (2004) The assessment of older driver's capabilities: A review of the literature, A. Arbor, editor. University of Michigan Transportation Research Institute

23. Seidler RD et al (2010) Motor control and aging: links to age-related brain structural, functional, and biochemical effects. Neurosci Biobehav Rev 34(5): 721-733

24. Voelcker-Rehage C (2008) Motor-skill learning in older adults-a review of studies on age-related differences. Eur Rev Aging Phys Act 5(1):5-16

25. Raw RK et al (2012) Movement control in older adults: does old age mean middle of the road? J Exp Psychol Hum Percept Perform 38(3):735-745

26. Head D et al (2002) Age-related differences in the course of cognitive skill acquisition: the role of regional cortical shrinkage and cognitive resources. Psychol Aging 17(1):72-84

27. Salthouse TA (2005) Relations between cognitive abilities and measures of executive functioning. Neuropsychology 19(4):532-545

28. Porter BE (2011) Chapter 31 - Enforcement. In: Porter BE (ed) Handbook of Traffic Psychology. Academic Press, San Diego, pp 441-453

29. Freund B, Smith P (2011) Chapter 24 - Older Drivers. In: Porter BE (ed) Handbook of Traffic Psychology. Academic Press, San Diego, pp 339-351

30. Baumann M, Krems JF (2007) Situation Awareness and Driving: A Cognitive Model. In: Cacciabue PC (ed) Modelling Driver Behaviour in Automotive Environments. Springer, London, pp 253-265

31. Krems J, Baumann MK (2009) Driving and Situation Awareness: A Cognitive Model of Memory-Update Processes. In: Kurosu M (ed) Human Centered Design. Springer Berlin, Heidelberg, pp 986-994

32. McGwin JG, Brown DB (1999) Characteristics of traffic crashes among young, middle-aged, and older drivers. Accid Anal Prev 31(3):181-198

33. Hirokazu A et al (2010) Cognitive Study on Driver's Behaviour by Vehicle Trajectory and Eye Movement in Virtual Environment. In: Advances in Cognitive Ergonomics. CRC Press, pp 408-417

34. Justiss MD (2005) Development of a behind-the-wheel driving performance assessment for older adults. University of Florida, Ann Arbor, p 160

35. Coughlin JF, Reimer B, Mehler B (2011) Monitoring, managing, and motivating driver safety and well-being. IEEE Pervasive Computing 10(3):14-21

36. Dodge S et al (2016) Analysis of movement data. Int J Geogr Inf Sci 30(5): 825-834

37. Gugerty $\amalg$ (1997) Situation awareness during driving: explicit and implicit knowledge in dynamic spatial memory. J Exp Psychol Appl 3(1):42-66

38. Underwood G, Crundall D, Chapman P (2011) Driving simulator validation with hazard perception. Transport Res F: Traffic Psychol Behav 14(6):435-446

39. Endsley MR (1995) Toward a theory of situation awareness in dynamic systems. Hum Factors: The Journal of the Human Factors and Ergonomics Society 37(1):32-64

40. Mcllvaine Parsons H (1976) Caution behavior and its conditioning in driving. Hum Factors 18(4):397-407

41. Fuller R (1984) A conceptualization of driving behaviour as threat avoidance. Ergonomics 27(11):1139-1155

42. Fuller R (2011) Chapter 2 - Driver Control Theory: From Task Difficulty Homeostasis to Risk Allostasis. In: Porter BE (ed) Handbook of Traffic Psychology. Academic Press, San Diego., pp 13-26

43. Summala H (2007) Towards understanding motivational and emotional factors in driver behaviour: Comfort through satisficing. In: Modelling Driver Behaviour in Automotive Environments: Critical Issues in Driver Interactions with Intelligent Transport Systems, pp 189-207

44. Smith EE (1968) Choice reaction time: an analysis of the major theoretical positions. Psychol Bull 69(2):77-110

45. Voelcker-Rehage C (2008) Motor-skill learning in older adults-a review of studies on age-related differences. Eur Rev Aging Phys Act 5(1):5-16

46. Rakotonirainy A (2005) Design of context-aware systems for vehicles using complex system paradigms. In: CEUR Workshop Proceedings

47. Classen S, Awadzi KD, Mkanta WW (2008) Person-vehicle-environment interactions predicting crash-related injury among older drivers. Am 」 Occup Ther 62(5):580-587

48. Clarke D et al (2010) Older drivers' road traffic crashes in the UK. Accid Anal Prev 42(4):1018-1024

49. Marmeleira J et al (2012) Associations of physical activity with drivingrelated cognitive abilities in older drivers: an exploratory Study1,2. Percept Mot Skills 115(2):521-533

50. Charlton J et al (2006) Characteristics of older drivers who adopt selfregulatory driving behaviours. Transportation research. Part F, Traffic psychology and behaviour 9(5):363-373
51. Romoser MRE et al (2005) The use of a driving simulator to asses senior driver performance: increasing situational awareness through post-drive one-on-one advisement. In: Third International Driving Symposium on Human Factors in driver Assessment. Training and Vehicle Design, Rockport (ME)

52. Michon J (1985) A Critical View of Driver Behavior Models: What Do We Know, What Should We Do? In: Evans L, Schwing R (eds) Human Behavior and Traffic Safety. Springer, US, pp 485-524

53. Glaser, S., et al. 2007 An Integrated Driver-Vehicle-Environment (I-DVE) model to assess crash risks. The Meeting Planners

54. Beery K (2004) Beery VMl-administration, scoring and teaching manual. NCS Pearson Inc., Minneapolis

55. Hong I, Kurihara T, Iwasaki M (2008) Older drivers' perceptions, responses, and driving behaviours during complex traffic conditions at a signalized intersection. Proceedings of the Institution of Mechanical Engineers, Part D: Journal of Automobile Engineering 222(11):2063-2076

56. Yarkoni T (2012) Psychoinformatics: new horizons at the Interface of the psychological and computing sciences. Curr Dir Psychol Sci 21(6):391-397

57. Markowetz A et al (2014) Psycho-informatics: big data shaping modern psychometrics. Med Hypotheses 82(4):405-411

58. Dörrzapf $L$ et al (2015) Urban emotions - An interdisciplinary interface between geoinformatics and urban planning. gis.Science - Die Zeitschrift fur Geoinformatik 1:11-19

59. Sun QC et al (2018) Driving manoeuvre during lane maintenance in older adults: associations with neuropsychological scores. Transport Res F: Traffic Psychol Behav 53

60. Anderson SW et al (2012) Neuropsychological assessment of driving safety risk in older adults with and without neurologic disease. J Clin Exp Neuropsychol 34(9):895-905

61. Bieliauskas LA (2005) Neuropsychological assessment of geriatic driving competence. Brain Inj 19(3):221-226

62. Sun $Q$ et al (2017) Validating the efficacy of GPS tracking vehicle movement for driving behaviour assessment. Travel Behaviour and Society 6:32-43

63. Poole A, Ball LJ (2006) Eye Tracking in $\mathrm{HCl}$ and Usability Research. In: Claude $G$ (ed) Encyclopedia of Human Computer Interaction. IGl Global, Hershey, PA, USA, pp 211-219

64. Tatler, B.W., Eye Movements from Laboratory to Life, in Current Trends in Eye Tracking Research, M. Horsley, et al., Editors. 2014, Springer International Publishing. p. 17-35

65. Underwood $\mathrm{G}$ et al (2003) Visual attention while driving: sequences of eye fixations made by experienced and novice drivers. Ergonomics 46(6):629-646

66. Lee JD (2008) Fifty years of driving safety research. Hum Factors 50(3):521-528

67. Dukic T, Broberg T (2012) Older drivers' visual search behaviour at intersections. Transport Res F: Traffic Psychol Behav 15(4):462-470

68. Falkmer $T$ et al (2008) Fixation identification in centroid versus start-point modes using eye-tracking data. Percept Mot Skills 106(3):710-724

69. Sun Q et al (2017) Pursuing Precise Vehicle Movement Trajectory in Urban Residential Area Using Multi-GNSS RTK Tracking. Trans Res Procedia 25C 2356-2371

70. Sun QC et al (2018) Towards Unpacking Older Drivers' Visual-motor Coordination: A Gaze-based Integrated Driving Assessment. Accid Anal Prev 113:85-96

71. Sun QC et al (2018) Unpacking older drivers' mobility at roundabouts: their visual-motor coordination through driver-vehicle-environment interactions. Int J Sustainable Transportation

72. Fitzsimmons EJ et al (2012) Analyses of vehicle trajectories and speed profiles along horizontal curves. J Transportation Safety \& Security 5(3):187-207

73. Sun QC et al (2018) Unpacking older Drivers' manoeuvre at intersections: their visual-motor coordination and the underlying neuropsychological mechanisms. Transport Res F: Traffic Psychol Behav 58:11-18.

74. Sun $Q$ et al (2016) Assessing drivers' visual-motor coordination using eye tracking, GNSS and GIS: a spatial turn in driving psychology. J Spat Sci 61(2):299-316

75. Sun Q et al (2016) Investigating the Spatial Pattern of Older Drivers' Eye Fixation Behaviour and Associations with Their Visual Capacity. J Eye Mov Res 9:6

76. Nunes A, Reimer B, Coughlin JF (2018) People must retain control of autonomous vehicles. Nature 556:169-171

77. Zhu X, Tang S (2015) Autonomous vehicle: from a cognitive perspective. In: Multimedia, Communication and Computing Application: Proceedings of the 2014 International Conference on Multimedia, Communication and Computing Application (MCCA 2014), Xiamen, China, October 16-17, 2014. CRC Press 